\title{
Zero cycles on the moduli space of curves
}

\author{
Rahul Pandharipande and Johannes Schmitt
}

\begin{abstract}
While the Chow groups of 0-dimensional cycles on the moduli spaces of Deligne-Mumford stable pointed curves can be very complicated, the span of the 0-dimensional tautological cycles is always of rank 1 . The question of whether a given moduli point $\left[C, p_{1}, \ldots, p_{n}\right] \in \overline{\mathcal{M}}_{g, n}$ determines a tautological 0 -cycle is subtle. Our main results address the question for curves on rational and $K 3$ surfaces. If $C$ is a nonsingular curve on a nonsingular rational surface of positive degree with respect to the anticanonical class, we prove $\left[C, p_{1}, \ldots, p_{n}\right]$ is tautological if the number of markings does not exceed the virtual dimension in Gromov-Witten theory of the moduli space of stable maps. If $C$ is a nonsingular curve on a $K 3$ surface, we prove $\left[C, p_{1}, \ldots, p_{n}\right]$ is tautological if the number of markings does not exceed the genus of $C$ and every marking is a Beauville-Voisin point. The latter result provides a connection between the rank 1 tautological 0 -cycles on the moduli of curves and the rank 1 tautological 0 -cycles on $K 3$ surfaces.

Several further results related to tautological 0-cycles on the moduli spaces of curves are proven. Many open questions concerning the moduli points of curves on other surfaces (Abelian, Enriques, general type) are discussed.
\end{abstract}

Keywords. Chow groups; Moduli spaces of curves; Tautological rings

2020 Mathematics Subject Classification. 14C25; $14 \mathrm{H} 10$

[Français]

Zéro cycles sur l'espace de modules des courbes

Résumé. Alors que les groupes de Chow des zéro-cycles sur les espaces de modules de Deligne-Mumford des courbes stables pointés peuvent être très compliqués, le sous-groupe des zéro-cycles tautologiques est toujours de rang 1 . Savoir si un point de l'espace de modules $\left[C, p_{1}, \ldots, p_{n}\right] \in \overline{\mathcal{M}}_{g, n}$ détermine un zéro-cycle tautologique est une question subtile. Nos résultats principaux répondent à cette question pour les courbes sur les surfaces rationnelles et les surfaces $K 3$. Si $C$ est une courbe lisse sur une surface rationnelle lisse, de degré anticanonique positif, nous démontrons que $\left[C, p_{1}, \ldots, p_{n}\right]$ est tautologique si le nombre de points marqués n'excède pas la dimension virtuelle de l'espace de modules des applications stables en théorie de Gromov-Witten. Si $C$ est une courbe lisse sur une surface $K 3,\left[C, p_{1}, \ldots, p_{n}\right]$ est tautologique si le nombre de points marqués n'excède pas le genre de $C$ et si tout marquage est un point de Beauville-Voisin. Ce dernier résultat fournit une connexion entre les zéro-cycles sur l'espace de modules des courbes et les zéro-cycles tautologiques sur les surfaces $K 3$.

Plusieurs autres résultats reliés aux zéros-cycles tautologiques sur les espaces de modules de courbes sont établis et nous discutons de nombreuses questions ouvertes concernant les points correspondants aux courbes sur d'autres surfaces (abéliennes, d'Enriques, de type général) font l'objet de discussion.

Received by the Editors on June 26, 2019, and in final form on June 3, 2020.

Accepted on July 23, 2020.

Rahul Pandharipande and Johannes Schmitt

Departement Mathematik, ETH Zürich

e-mail: rahul@math.ethz.ch and johannes.schmitt@math.ethz.ch

R.P. was supported by SNF-200020-182181, ERC-2017-AdG-786580-MACI, SwissMAP, and the Einstein Stiftung. J.S. was supported by SNF-200020-162928 and ERC-2017-AdG-786580-MACI, by the SNF early postdoc mobility grant 184245 and wants to thank the Max Planck Institute for Mathematics in Bonn for its hospitality. This project has received funding from the European Research Council (ERC) under the European Union Horizon 2020 research and innovation programme (grant agreement No 786580).

(C) by the author(s)

This work is licensed under http://creativecommons.org/licenses/by-sa/4.0/ 


\section{Contents}

1. Introduction. . . . . . . . . . . . . . . . . . . . . . . . . . . . . . . . 3

1.1. Moduli of curves . . . . . . . . . . . . . . . . . . . . . . . . . . . . . 3

1.2. 0 -cycles in the tautological ring . . . . . . . . . . . . . . . . . . . . . . . 3

1.3. Tautological points . . . . . . . . . . . . . . . . . . . . . . . . . . . . 5

1.4. Curves on surfaces. . . . . . . . . . . . . . . . . . . . . . . . . . . . . 5

1.5. Further results on tautological 0 -cycles. . . . . . . . . . . . . . . . . . . . . 7

1.6. $T$-numbers for $K 3$ surfaces . . . . . . . . . . . . . . . . . . . . . . . . . . . 8

1.7. Plan of the paper . . . . . . . . . . . . . . . . . . . . . . . . . . . . . . . . 8

1.8. Acknowledgements . . . . . . . . . . . . . . . . . . . . . . . . . . . . 8

2. Basic results about cycles and curves . . . . . . . . . . . . . . . . . . . . . . 8

3. Rational surfaces . . . . . . . . . . . . . . . . . . . . . . . . . . . . . . 10

3.1. Proof of Theorem 1.4 . . . . . . . . . . . . . . . . . . . . . . . . . . . . 10

3.2. Variations . . . . . . . . . . . . . . . . . . . . . . . . . . . . 12

4. K3 surfaces . . . . . . . . . . . . . . . . . . . . . . . . . . . . . . . . 13

4.1. Beauville-Voisin classes . . . . . . . . . . . . . . . . . . . . . . . . . . . 13

4.2. Proof of Theorem 1.5 . . . . . . . . . . . . . . . . . . . . . . . . . . . . 13

4.3. Quotients . . . . . . . . . . . . . . . . . . . . . . . . 15

5. Other surface geometries . . . . . . . . . . . . . . . . . . . . . . . . . 17

5.1. Enriques surfaces . . . . . . . . . . . . . . . . . . . . . . . . . . . . . 17

5.2. Abelian surfaces. . . . . . . . . . . . . . . . . . . . . . . . . . . . . . 18

5.3. Surfaces of general type. . . . . . . . . . . . . . . . . . . . . . . . . . . 19

6. Cyclic covers . . . . . . . . . . . . . . . . . . . . . . . . . . . . . . . 19

7. Summing to tautological cycles . . . . . . . . . . . . . . . . . . . . . . . . 21

7.1. Existence . . . . . . . . . . . . . . . . . . . . . . . . . . . . . . . . 21

7.2. Minimality . . . . . . . . . . . . . . . . . . . . . . . . . . 22

References. . . . . . . . . . . . . . . . . . . . . . . . . . . . 24 


\section{Introduction}

\subsection{Moduli of curves}

Let $\left(C, p_{1}, \ldots, p_{n}\right)$ be a Deligne-Mumford stable curve of genus $g$ with $n$ marked points defined over $\mathbb{C}$. Let

$$
\left[C, p_{1}, \ldots, p_{n}\right] \in \overline{\mathcal{M}}_{g, n}
$$

be the associated moduli point in the moduli space. ${ }^{1}$ As a Deligne-Mumford stack, $\overline{\mathcal{M}}_{g, n}$ is nonsingular, irreducible, and of complex dimension $3 g-3+n$. Though the moduli spaces $\overline{\mathcal{M}}_{g, n}$ can be irrational and complicated, their study has been marked by the discovery of beautiful mathematical structures.

Fundamental to the geometry of the moduli spaces of stable pointed curves are three basic types of morphisms:

(i) forgetful morphisms

$$
p: \overline{\mathcal{M}}_{g, n+1} \rightarrow \overline{\mathcal{M}}_{g, n}
$$

defined by dropping a marking,

(ii) irreducible boundary morphisms

$$
q: \overline{\mathcal{M}}_{g-1, n+2} \rightarrow \overline{\mathcal{M}}_{g, n}
$$

defined by identifying two markings to create a node,

(iii) reducible boundary morphisms

$$
r: \overline{\mathcal{M}}_{g_{1}, n_{1}+1} \times \overline{\mathcal{M}}_{g_{2}, n_{2}+1} \rightarrow \overline{\mathcal{M}}_{g, n},
$$

where $n=n_{1}+n_{2}$ and $g=g_{1}+g_{2}$, defined by identifying the markings of separate pointed curves. Following [FP05, Section 0.1], the tautological rings ${ }^{2}$

$$
R^{*}\left(\overline{\mathcal{M}}_{g, n}\right) \subset A^{*}\left(\overline{\mathcal{M}}_{g, n}\right)
$$

are defined as the smallest system of $\mathbb{Q}$-subalgebras (with unit) closed under push-forward by all morphisms (i)-(iii). We denote the group of tautological $k$-cycles by

$$
R_{k}\left(\overline{\mathcal{M}}_{g, n}\right)=R^{3 g-3+n-k}\left(\overline{\mathcal{M}}_{g, n}\right) .
$$

For an introduction to the current study of tautological classes, we refer the reader to [FP13, Pan18].

\subsection{0 -cycles in the tautological ring}

Whenever the moduli space $\overline{\mathcal{M}}_{g, n}$ is rationally connected, we have

$$
A_{0}\left(\overline{\mathcal{M}}_{g, n}\right) \stackrel{\simeq}{=} \text {. }
$$

Rational connectedness is known at least in the cases appearing in Figure 1. For genus 23 and higher, $\overline{\mathcal{M}}_{g, n}$ is never rationally connected.

\begin{tabular}{|c|c|c|c|c|c|c|c|c|c|c|c|c|c|c|c|c|}
\hline$g$ & 0 & 1 & 2 & 3 & 4 & 5 & 6 & 7 & 8 & 9 & 10 & 11 & 12 & 13 & 14 & 15 \\
\hline$n_{\max }$ & $\infty$ & 10 & 12 & 14 & 15 & 12 & 15 & 11 & 8 & 9 & 3 & 10 & 1 & 0 & 2 & 0 \\
\hline
\end{tabular}

Figure 1. $\overline{\mathcal{M}}_{g, n}$ is rationally connected for $n \leq n_{\max }$, see [Ben14, BV05, CF07, Far09, Log03, Ver05].

\footnotetext{
${ }^{1}$ Stability requires $2 g-2+n>0$ which we always impose when we write $\overline{\mathcal{M}}_{g, n}$.

${ }^{2}$ Chow groups will be taken with $\mathbb{Q}$-coefficients unless explicitly stated otherwise.
} 
On the other hand, the Chow groups of 0 -cycles are of infinite rank as $\mathbb{Q}$-vector spaces at least in the following genus 1 and 2 cases (due to the existence ${ }^{3}$ of holomorphic $p$-forms):

$$
A_{0}\left(\overline{\mathcal{M}}_{1, n \geq 11}\right), \quad A_{0}\left(\overline{\mathcal{M}}_{2, n \geq 14}\right) \text {. }
$$

Moreover, such forms ${ }^{4}$ and infinite ranks are expected in the following genus 3 and 4 cases:

$$
A_{0}\left(\overline{\mathcal{M}}_{3, n \geq 15}\right), \quad A_{0}\left(\overline{\mathcal{M}}_{4, n \geq 16}\right) \text {. }
$$

While the data is insufficient for a general prediction, the following speculation would not be surprising.

Speculation 1.1. For $g \geq 1$, the Chow group $A_{0}\left(\overline{\mathcal{M}}_{g, n}\right)$ is of infinite rank except for finitely many $(g, n)$.

On the other hand, the group $R_{0}\left(\overline{\mathcal{M}}_{g, n}\right)$ of tautological 0 -cycles is much better behaved. The following result was proven by Graber and Vakil in [GV01] and also in [FP05, HL97].

Proposition 1.2. For all $(g, n)$, we have $R_{0}\left(\overline{\mathcal{M}}_{g, n}\right) \stackrel{\simeq}{=} \mathbb{Q}$.

Since the proof is so short (and depends only upon structural properties of tautological classes), we present the argument here. ${ }^{5}$ Consider the moduli space $\overline{\mathcal{M}}_{0,2 g+n}$ together with the boundary morphism

$$
\iota: \overline{\mathcal{M}}_{0,2 g+n} \rightarrow \overline{\mathcal{M}}_{g, n}
$$

defined by pairing the first $2 g$ markings to create $g$ nodes. Since $\overline{\mathcal{M}}_{0,2 g+n}$ is a rational variety,

$$
R_{0}\left(\overline{\mathcal{M}}_{0,2 g+n}\right)=A_{0}\left(\overline{\mathcal{M}}_{0,2 g+n}\right) \stackrel{\simeq}{=} .
$$

Therefore, all the moduli points in the image of $\iota$ are tautological and span a $\mathbb{Q}$-subspace of $R_{0}\left(\overline{\mathcal{M}}_{g, n}\right)$ of rank 1 . We will prove that the span equals $R_{0}\left(\overline{\mathcal{M}}_{g, n}\right)$.

Using the additive generators of the tautological ring constructed in [GP03, Appendix], we need only consider 0 -cycles on $\overline{\mathcal{M}}_{g, n}$ which are of a special form. The strata of $\overline{\mathcal{M}}_{g, n}$ are indexed by stable graphs $\Gamma$ of genus $g$ with $n$ markings,

We need only consider 0 -cycles

$$
\iota_{\Gamma}: \overline{\mathcal{M}}_{\Gamma} \rightarrow \overline{\mathcal{M}}_{g, n}
$$

$$
\iota_{\Gamma *}\left[\prod_{v \in \operatorname{Vert}(\Gamma)} P(v)\right] \in R_{0}\left(\overline{\mathcal{M}}_{g, n}\right),
$$

where $P(v)$ is a monomial in $\psi$ and $\mathcal{\kappa}$ classes on the moduli space $\overline{\mathcal{M}}_{g(v), n(v)}$ associated to the vertex $v$. Let $\operatorname{deg}(P(v))$ be the degree of the vertex class. Using the Getzler-Ionel vanishing in the strong form proven ${ }^{6}$ in [FP05, Proposition 2], we can impose the following additional restriction on (1.1):

$$
g(v)>0 \Rightarrow \operatorname{deg}(P(v))<g-\delta_{0, n(v)} .
$$

Suppose we have a vertex $v$ of $\Gamma$ with $g(v)>0$. Using the vertex stability condition $2 g(v)-2+n(v)>0$, we deduce

But then we obtain

$$
g(v)-\delta_{0, n(v)} \leq 3 g(v)-3+n(v)
$$

$$
\operatorname{deg}(P(v))<3 g(v)-3+n(v)
$$

which is impossible since (1.1) is a 0 -cycle. Therefore, we must have $g(v)=0$ for all $v \in \operatorname{Vert}(\Gamma)$.

\footnotetext{
${ }^{3}$ By results of Mumford and Srinivas (see [Mum68, Ro72, Sri87] and [GV01, Remark 1.1]), the existence of a holomorphic $p$-form for $p \geq 1$ forces $A_{0}\left(\overline{\mathcal{M}}_{g, n}\right)$ to have infinite rank. Constructions of such forms in $g=1$ and $g=2$ are well-known, see [FP13].

${ }^{4}$ There are no written proofs for the genus 3 and 4 claims, but these expectations, based on geometric calculations, have been communicated to us by Faber (in genus 3) and Farkas (in genus 4).

${ }^{5}$ We follow the path of the proof [FP05, HL97]. See [FP05, Section 4] and [HL97, Section 5.1].

${ }^{6}$ See [CJWZ17] for a much more effective approach to the boundary terms than provided by the argument of [FP05].
} 
The 0 -cycle (1.1) is now easily seen to be in the image of

$$
\iota_{*}: R_{0}\left(\overline{\mathcal{M}}_{0,2 g+n}\right) \rightarrow R_{0}\left(\overline{\mathcal{M}}_{g, n}\right) .
$$

We conclude that the push-forward (1.3) is surjective.

\subsection{Tautological points}

Our central question here is how to decide whether a given moduli point

$$
\left[C, p_{1}, \ldots, p_{n}\right] \in \overline{\mathcal{M}}_{g, n}
$$

determines a tautological 0 -cycle.

While our focus is on the geometry of $C$, there is an interesting connection to arithmetic: Bloch and Beilinson have conjectured ${ }^{7}$ that for a nonsingular proper variety $X$ defined over $\overline{\mathbb{Q}}$, the complex Abel-Jacobi map

$$
\Phi_{k, \mathbb{Q}}: A_{\mathrm{hom}}^{k}(X / \overline{\mathbb{Q}})_{\mathbb{Q}} \rightarrow J^{k}(X(\mathbb{C}))_{\mathbb{Q}}
$$

to the intermediate Jacobian $J^{k}(X(\mathbb{C})$ ) is injective (after tensoring with $\mathbb{Q}$ ). The map above factors through the usual Abel-Jacobi map of $X(\mathbb{C})$, and the image of $A_{\text {hom }}^{k}(X / \overline{\mathbb{Q}})_{\mathbb{Q}}$ in $A_{\text {hom }}^{k}(X(\mathbb{C}))_{\mathbb{Q}}$ is the set of $k$-cycles in $X(\mathbb{C})$ defined over $\overline{\mathbb{Q}}$ which are homologous to 0 . If the Bloch-Beilinson conjecture holds for

$$
X=\overline{\mathcal{M}}_{g, n} \text {, }
$$

the map

$$
\Phi_{3 g-3+n, \mathbb{Q}}: A_{0}\left(\overline{\mathcal{M}}_{g, n}\right)_{\text {hom }} \rightarrow \operatorname{Alb}\left(\overline{\mathcal{M}}_{g, n}\right) \otimes \mathbb{Q}
$$

would be injective on the set of 0 -cycles defined over $\overline{\mathbb{Q}}$. But since $\overline{\mathcal{M}}_{g, n}$ is simply connected [BP00, Proposition 1.1], the Albanese variety is trivial. Since a tautological class in $A_{0}\left(\overline{\mathcal{M}}_{g, n}\right)$ can be represented by a curve defined over $\overline{\mathbb{Q}}$, we would obtain the following consequence.

Speculation 1.3. If the pointed curve $\left(C, p_{1}, \ldots, p_{n}\right)$ is defined over $\overline{\mathbb{Q}}$, then the associated moduli point in $A_{0}\left(\overline{\mathcal{M}}_{g, n}\right)$ is tautological.

A first step in the study of Speculation 1.3 is perhaps to use Belyi's Theorem to express the curve as a Hurwitz covering

$$
C \rightarrow \mathbb{P}^{1}
$$

ramified only over 3 points of $\mathbb{P}^{1}$. Unfortunately, there has not been much progress in the direction of Speculation 1.3. However, we will present a result about cyclic covers of $\mathbb{P}^{1}$ in Section 6.

\subsection{Curves on surfaces}

Instead of studying the moduli points of special Hurwitz covers of $\mathbb{P}^{1}$, our main results here concern the moduli points of curves on special surfaces.

\section{Rational surfaces}

Let $S$ be a nonsingular projective rational surface over $\mathbb{C}$, and let $C \subset S$ be an irreducible nonsingular curve of genus $g$. The virtual dimension in Gromov-Witten theory of the moduli space of stable maps $\overline{\mathcal{M}}_{g}(S,[C])$ is given by the following formula

$$
\operatorname{vdim} \overline{\mathcal{M}}_{g}(S,[C])=\int_{[C]} c_{1}(S)+g-1 .
$$

Our first result gives a criterion for curves on rational surfaces in terms of the virtual dimension.

\footnotetext{
${ }^{7}$ See [Be87, Blo85] for the original papers by Bloch and Beilinson and [Jan90] for a detailed account. See [Jan90, Conjecture 9.12] and the remark thereafter for the particular form of the conjecture that we have used.
} 
Theorem 1.4. Let $C \subset S$ be an irreducible nonsingular curve of genus $g$ on a nonsingular rational surface satisfying $\int_{[C]} c_{1}(S)>0$. Let $p_{1}, \ldots, p_{n} \in C$ be distinct points. If

$$
n \leq \operatorname{vdim} \overline{\mathcal{M}}_{g}(S,[C]),
$$

then $\left[C, p_{1}, \ldots, p_{n}\right] \in \overline{\mathcal{M}}_{g, n}$ determines a tautological 0 -cycle in $R_{0}\left(\overline{\mathcal{M}}_{g, n}\right)$.

For Theorem 1.4, we always assume $(g, n)$ is in the stable range

$$
2 g-2+n>0 \text {. }
$$

If positivity

$$
\int_{[C]} c_{1}(S)>0
$$

holds, then Theorem 1.4 can be applied with $n=0$ to obtain

$$
[C] \in R_{0}\left(\overline{\mathcal{M}}_{g}\right) \text {. }
$$

In case $S$ is toric, positivity (1.4) always holds for nonsingular curves of genus $g \geq 1$ since there exists an effective toric anticanonical divisor with affine complement. Whether positivity (1.4) can be avoided in Theorem 1.4 is an interesting question. ${ }^{8}$

As an example, consider a nonsingular curve

$$
C_{4} \subset \mathbb{P}^{1} \times \mathbb{P}^{1}
$$

of genus 4 and bidegree $(3,3)$. Positivity $(1.4)$ holds, and the virtual dimension here is 15 , so all moduli points

$$
\left[C_{4}, p_{1}, \ldots, p_{15}\right] \in \overline{\mathcal{M}}_{4,15}
$$

are tautological. Since the general curve of genus 4 is of the form $C_{4}$, but not all points of $\overline{\mathcal{M}}_{4,16}$ are expected to be tautological, the virtual dimension bound on $n$ in Theorem 1.4 should not have room for improvement here.

\section{K3 surfaces}

Let $S$ be a nonsingular projective $K 3$ surface over $\mathbb{C}$. Unlike the case of a rational surface, the Chow group $A_{0}(S, \mathbb{Z})$ of 0 -cycles of $S$ is very complicated. However, there is a beautiful rank 1 subspace

$$
\mathrm{BV} \subset A_{0}(S, \mathbb{Z})
$$

spanned by points lying on rational curves of $S$. Following [BV04], define $p \in S$ to be a Beauville-Voisin point if $[p] \in \mathrm{BV}$.

Let $C \subset S$ be an irreducible nonsingular curve of genus $g$. The virtual dimension of the moduli space of stable maps $\overline{\mathcal{M}}_{g}(S,[C])$ is now

$$
\operatorname{vdim} \overline{\mathcal{M}}_{g}(S,[C])=g-1 .
$$

Important for us, however, will be the reduced virtual dimension $g$. Our second result gives a criterion for curves on $K 3$ surfaces.

Theorem 1.5. Let $C \subset S$ be an irreducible nonsingular curve of genus $g$ on a K3 surface. Let

$$
p_{1}, \ldots, p_{n} \in C
$$

be distinct Beauville-Voisin points of $S$. If $n \leq g$, then $\left[C, p_{1}, \ldots, p_{n}\right] \in \overline{\mathcal{M}}_{g, n}$ determines a tautological 0 -cycle in $R_{0}\left(\overline{\mathcal{M}}_{g, n}\right)$.

\footnotetext{
${ }^{8}$ The issue is not unrelated to the Harbourne-Hartshorne conjecture and will be discussed in Section 3.2.
} 
For example, consider a nonsingular curve of genus 11

$$
\left(C_{11}, p_{1}, \ldots, p_{11}\right) \subset S
$$

in a primitive class on a $K 3$ surface $S$ with 11 distinct points. By Theorem 1.5,

$$
\left[C_{11}, p_{1}, \ldots, p_{11}\right] \in R_{0}\left(\overline{\mathcal{M}}_{11,11}\right)
$$

in case all the points $p_{i}$ are Beauville-Voisin. By the Mukai correspondence [Muk96], we can obtain the general moduli point of $\overline{\mathcal{M}}_{11,11}$ by varying the data (1.5) in the moduli space of polarized $K 3$ surfaces of genus 11 with 11 points. Since $\overline{\mathcal{M}}_{11,11}$ is of Kodaira dimension 19 by [FV18, Theorem 5.1], the Chow group of 0 -cycles is expected (but not known) to be complicated. In particular, the general moduli point of $\overline{\mathcal{M}}_{11,11}$ is not expected to be tautological. The geometry of $K 3$ surfaces in genus 11 therefore suggests that a condition on the points is necessary.

The condition of Theorem 1.5 exactly links the rank 1 Beauville-Voisin subspace

$$
\mathrm{BV} \subset A_{0}(S, \mathbb{Z})
$$

to the rank 1 tautological subspace

$$
R_{0}\left(\overline{\mathcal{M}}_{g, n}\right) \subset A_{0}\left(\overline{\mathcal{M}}_{g, n}\right)
$$

\section{Other surfaces}

Since every nonsingular curve lies on a nonsingular algebraic surface, results along the lines of Theorems 1.4 and 1.5 will always require special surface geometries. For nonsingular curves lying on Enriques and Abelian surfaces, we hope for results parallel to those in the rational and $K 3$ surface cases. However, the questions are, at the moment, open. For the Enriques surfaces, there is a clear path, but the argument depends upon currently open questions about the nonemptiness of certain Severi varieties. For Abelian surfaces, the matter appears more subtle (and there is no obvious line of argument that we can see).

For surfaces of general type, canonical curves play a very special role from the perspective of GromovWitten and Seiberg-Witten theories. A natural question to ask is whether a nonsingular canonical curve on a surface of general type always determine a tautological 0-cycle. We expect new strategies will be required to resolve such questions in the general type case.

\subsection{Further results on tautological 0 -cycles}

We have seen that a moduli point

$$
\left[C, p_{1}, \ldots, p_{n}\right] \in \overline{\mathcal{M}}_{g, n}
$$

need not determine a tautological 0 -cycle. We can measure how far away from tautological moduli points of $\overline{\mathcal{M}}_{g, n}$ are by considering sums. Let

$$
T(g, n) \in \mathbb{Z}_{>0}
$$

be the smallest number satisfying the following condition: for every point $Q_{1} \in \overline{\mathcal{M}}_{g, n}$, there exist $T(g, n)-1$ other points $Q_{2}, \ldots, Q_{T(g, n)} \in \overline{\mathcal{M}}_{g, n}$ which together have a tautological sum

$$
\left[Q_{1}\right]+\left[Q_{2}\right]+\cdots+\left[Q_{T(g, n)}\right] \in R_{0}\left(\overline{\mathcal{M}}_{g, n}\right) .
$$

An easy proof of the existence of $T(g, n)$ is given in Section 7. Finding good bounds for $T(g, n)$ appears much harder. Our main result here states that the growth of $T(g, n)$ for fixed $g$ as $n \rightarrow \infty$ is at most linear in $n$. Can better asymptotics be found? For example, could $T(g, n)$ for fixed $g$ be bounded independent of $n$ ? 


\section{6. $T$-numbers for $K 3$ surfaces}

For comparison, we can consider the parallel question for a $K 3$ surface $S$, namely: what is the smallest positive integer $T$ such that for any given $p \in S$ we find $q_{2}, \ldots, q_{T} \in S$ such that the sum

$$
[p]+\left[q_{2}\right]+\cdots+\left[q_{T}\right] \in A_{0}(S, \mathbb{Z})
$$

lies in the Beauville-Voisin subspace $\mathrm{BV} \subset A_{0}(S, \mathbb{Z})$ ?

On the one hand, we have $T \geq 2$, since $T=1$ would be the statement that for every $p \in S$ we have $[p] \in \mathrm{BV}$, a contradiction since $A_{0}(S, \mathbb{Z})$ is infinite-dimensional and spanned by the classes $[p]$. On the other hand, since we have families of elliptic curves which sweep out $S$, the given point $p$ must lie on a (possibly singular) genus 1 curve $E \subset S$. Let $R \subset S$ be a rational curve in an ample class. Since

$$
R \cap E \neq \emptyset,
$$

$E$ contains a Beauville-Voisin point $z \in E$. We can always solve the equation

$$
[p]+[q]=2[z] \in A_{0}(E, \mathbb{Z})
$$

for $q \in E$. We conclude that for any $p \in S$, there exists a $q \in S$ satisfying

$$
[p]+[q] \in \mathrm{BV} .
$$

The $T$-number for $K 3$ surfaces is therefore just 2.

The Hilbert scheme $S^{[n]}$ of $n$ points on $S$ also has a holomorphic form and a distinguished Beauville-Voisin subspace in $A_{0}\left(S^{[n]}, \mathbb{Z}\right)$. The holomorphic form shows that the $T$-number of $S^{[n]}$ is greater than 1 . Using families of elliptic curves on $S$, the $T$-number of $S^{[n]}$ is proven to be at most $n+1$ in the upcoming paper [SY], again a linear bound. Whether the $T$-number is exactly $n+1$ is an interesting question.

\subsection{Plan of the paper}

We start in Section 2 with basic results about cycles and curves which we will use throughout the paper. Theorem 1.4 for rational surfaces is proven in Section 3 and Theorem 1.5 for $K 3$ surfaces is proven in Section 4. Open questions for Enriques surfaces, Abelian surfaces, and surfaces of general type are discussed in Section 5. A result concerning cyclic covers of $\mathbb{C P}^{1}$ is proven in Section 6 . The paper ends with results about the number $T(g, n)$ in Section 7.

\subsection{Acknowledgements}

We thank C. Faber for contributing to our study of curves and G. Farkas for useful conversations about the birational geometry of moduli spaces. We thank A. Knutsen for discussions about Severi varieties of Enriques surfaces. Discussions with T. Bülles, A. Kresch, D. Petersen, U. Riess, J. Shen, and Q. Yin have played an important role. We thank the anonymous referee for many helpful comments, improving and clarifying our exposition. An early version of the results was presented at the workshop Hurwitz cycles on the moduli of curves at Humboldt Universität zu Berlin in February 2018.

\section{Basic results about cycles and curves}

We start by recalling the following useful (and well-known) result about families of algebraic cycles, see [Voil5, Proposition 2.4].

Proposition 2.1. Let $\pi: \mathcal{X} \rightarrow B$ be a flat morphism of algebraic varieties where $B$ is nonsingular of dimension $r$ and let $\mathcal{Z} \in A_{N}(\mathcal{X})$ be a cycle. Then, the set $B_{Z}$ of points $t \in B$ satisfying

$$
\mathcal{Z}_{t}=\left.\mathcal{Z}\right|_{\mathcal{X}_{t}}=0 \in A_{N-r}\left(\mathcal{X}_{t}\right)
$$

is a countable union of proper closed algebraic subsets of $B$. 
Proposition 2.2. Let $X \subset \overline{\mathcal{M}}_{g, n}$ be an irreducible algebraic set such that the generic point of $X$ is tautological. Then, every point of $X$ is tautological.

Proof. Consider the trivial family

$$
\pi: \overline{\mathcal{M}}_{g, n} \times \overline{\mathcal{M}}_{g, n} \rightarrow \overline{\mathcal{M}}_{g, n}
$$

defined by projection on the second factor. Let $\Delta \subset \overline{\mathcal{M}}_{g, n} \times \overline{\mathcal{M}}_{g, n}$ be the diagonal, and let $S$ be the section of $\pi$ determined by a fixed tautological point of $\overline{\mathcal{M}}_{g, n}$. By applying ${ }^{9}$ Proposition 2.1 to the relative 0 -cycle

$$
\mathcal{Z}=\Delta-S
$$

the set of points in $\bar{M}_{g, n}$ whose class is tautological is a countable union of closed algebraic sets. Since the generic point of $X$ is contained in this union, $X$ must also be contained.

Let $S$ be a nonsingular projective surface which is either rational or $K 3$. In both cases,

$$
\operatorname{Pic}(S)=H_{2}(S, \mathbb{Z}) \text {. }
$$

Let $L \in \operatorname{Pic}(S)$ be an effective divisor class. Let $|L|=\mathbb{P}\left(H^{0}(S, L)\right)$ be the associated linear system of divisors with hyperplane class $H \in A^{1}(|L|)$. There exists a natural Hilbert-Chow morphism

$$
c: \overline{\mathcal{M}}_{g, n}\left(S, c_{1}(L)\right) \rightarrow|L|,
$$

sending a stable map $\left(f:\left(C, p_{1}, \ldots, p_{n}\right) \rightarrow S\right)$ to the effective divisor $f_{*}[C]$.

In the stable range $2 g-2+n>0$, let

$$
\epsilon: \overline{\mathcal{M}}_{g, n}\left(S, c_{1}(L)\right) \rightarrow \overline{\mathcal{M}}_{g, n}
$$

be the natural forgetful morphism. Let

$$
\mathrm{ev}_{i}: \overline{\mathcal{M}}_{g, n}\left(S, c_{1}(L)\right) \rightarrow S
$$

be the evaluation map corresponding to the $i$ th marking.

Lemma 2.3. Let $S$ be a rational surface with $L \in \operatorname{Pic}(S)$. Let $C \subset S$ be a nonsingular irreducible curve of genus g contained in $|L|$. Assume

$$
\operatorname{dim}|L|=\operatorname{vdim} \overline{\mathcal{M}}_{g}(S,[C])=g-1+\int_{[C]} c_{1}(S) .
$$

Then, for $0 \leq n \leq \operatorname{vdim} \overline{\mathcal{M}}_{g}(S,[C])$ satisfying $2 g-2+n>0$ and pairwise distinct points $p_{1}, \ldots, p_{n} \in C$, we have

$$
\epsilon_{*}\left(c^{*} H^{\operatorname{dim}|L|-n} \cap \prod_{i=1}^{n} \operatorname{ev}_{i}^{*}\left[p_{i}\right] \cap\left[\overline{\mathcal{M}}_{g, n}(S,[C])\right]^{\mathrm{vir}}\right)=\left[C, p_{1}, \ldots, p_{n}\right]
$$

in $A_{0}\left(\overline{\mathcal{M}}_{g, n}\right)$.

Proof. We first prove the Lemma for general points

$$
p_{1}, \ldots, p_{n} \in C .
$$

For general points $p_{i}$, the set of curves in $|L|$ passing through the $p_{i}$ is a linear subspace $H_{1}$ of codimension $n$. We choose a complementary linear subspace $H_{2} \subset|L|$ of codimension $r-n$ satisfying

$$
H_{1} \cap H_{2}=\{[C]\} .
$$

Therefore, on $\overline{\mathcal{M}}_{g, n}(S,[C])$, the cycle $c^{*}\left[H_{2}\right] \cap \prod_{i=1}^{n} \mathrm{ev}_{i}^{*}\left[p_{i}\right]$ is supported on the point

$$
\left[\left(C, p_{1}, \ldots, p_{n}\right) \hookrightarrow S\right] \in \overline{\mathcal{M}}_{g, n}(S,[C])
$$

\footnotetext{
${ }^{9}$ We leave the standard movement of scheme results to stacks for the reader.
} 
Near the point $(2.3)$ in $\overline{\mathcal{M}}_{g, n}(S,[C])$, the map $\Phi=\left(c, \mathrm{ev}_{1}, \ldots, \mathrm{ev}_{n}\right)$ defines a local isomorphism ${ }^{10}$ to the incidence variety

$$
\mathcal{I}=\left\{\left(D, q_{1}, \ldots, q_{n}\right): D \in|L|, q_{1}, \ldots, q_{n} \in D\right\} \subset|L| \times S^{n} .
$$

Since near (2.3) $\mathcal{I}$ is nonsingular of dimension $\operatorname{dim}|L|+n$ and since this is the virtual dimension of $\overline{\mathcal{M}}_{g, n}(S,[C])$, the virtual fundamental class restricts to the standard fundamental class near (2.3). Since $H_{2} \times \prod_{i=1}^{n}\left[p_{i}\right]$ intersects $\mathcal{I}$ transversally in the point $\left([C], p_{1}, \ldots, p_{n}\right)$, we obtain the equality (2.2).

We finish the proof by going from the case of general points $p_{1}, \ldots, p_{n} \in C$ to the case of any pairwise distinct set of points. Consider the complement $B=C^{n} \backslash \Delta$ of the diagonals inside the product $C^{n}$. The difference of the two sides of equation (2.2) defines a natural cycle $\mathcal{Z}$ inside $\overline{\mathcal{M}}_{g, n} \times B$. For $b \in B$ general, we have

$$
\left.\mathcal{Z}\right|_{\overline{\mathcal{M}}_{g, n} \times\{b\}}=0 .
$$

By Proposition 2.1, the set of such $b$ is a countable union of closed algebraic sets, and so must be all of $B$.

For $S$ a nonsingular projective $K 3$ surface, we need a variant of Lemma 2.3 involving the reduced virtual fundamental class (see [BL00, MP13]).

Lemma 2.4. Let $S$ be a $K 3$ surface with $L \in \operatorname{Pic}(S)$. Let $C \subset S$ be a nonsingular irreducible curve of genus $g$ contained in $|L|$. Then for

$$
0 \leq n \leq g \text { satisfiying } 2 g-2+n>0
$$

and distinct points $p_{1}, \ldots, p_{n} \in C$, we have

$$
\epsilon_{*}\left(c^{*} H^{g-n} \cdot \prod_{i=1}^{n} \mathrm{ev}_{i}^{*}\left[p_{i}\right] \cap\left[\overline{\mathcal{M}}_{g, n}(S,[C])\right]^{\mathrm{red}}\right)=\left[C, p_{1}, \ldots, p_{n}\right]
$$

in $A_{0}\left(\overline{\mathcal{M}}_{g, n}\right)$.

Proof. Since $L=\mathcal{O}_{S}(C)$, the exact sequence

$$
0 \rightarrow H^{0}\left(S, \mathcal{O}_{S}\right) \rightarrow H^{0}\left(S, \mathcal{O}_{S}(C)\right) \rightarrow H^{0}\left(C, \mathcal{O}_{C}(C)\right) \rightarrow H^{1}\left(S, \mathcal{O}_{S}\right) \rightarrow \ldots
$$

together with the ranks

$$
h^{0}\left(C, \mathcal{O}_{C}(C)\right)=h^{0}\left(C, \omega_{C}\right)=g, \quad h^{1}\left(S, \mathcal{O}_{S}\right)=0
$$

shows $\operatorname{dim}|L|=g$. Hence, we have

$$
\operatorname{dim}|L|+n=g+n=\operatorname{dim}\left[\overline{\mathcal{M}}_{g, n}\left(S, c_{1}(L)\right)\right]^{\mathrm{red}} .
$$

The proof of Lemma 2.3 can then be exactly followed for the reduced class here to conclude the result.

\section{Rational surfaces}

\subsection{Proof of Theorem 1.4}

If $C$ is of genus $g=0$, Theorem 1.4 is trivial (since the moduli space $\overline{\mathcal{M}}_{0, n}$ is rational and all 0 -cycles are tautological). We will assume $g \geq 1$. The argument proceeds in three steps:

(1) We apply Lemma 2.3 to express the 0 -cycle

$$
\left[C, p_{1}, \ldots, p_{n}\right] \in A_{0}\left(\overline{\mathcal{M}}_{g, n}\right)
$$

in terms of a push-forward involving the virtual fundamental class of $\overline{\mathcal{M}}_{g, n}(S,[C])$.

(2) We deform the rational surface $S$ to a nonsingular projective toric surface $\widehat{S}$ over a base which is rationally connected.

\footnotetext{
${ }^{10}$ Since all the curves $D$ near $C$ are irreducible and nonsingular, the inverse map is well-defined.
} 
(3) We apply virtual localization [GP99] to the toric surface $\widehat{S}$ to conclude the desired class is tautological. Step 1. To apply Lemma 2.3, we must check the hypothesis

$$
\operatorname{dim}|L|=g-1+\int_{[C]} c_{1}(S),
$$

where $L=\mathcal{O}_{S}(C)$. Condition (3.1) is equivalent to $h^{0}(L)=g+\int_{[C]} c_{1}(S)$.

Since $C$ is nonsingular of genus $g$, the adjunction formula yields

$$
\left\langle[C],[C]-c_{1}(S)\right\rangle=2 g-2,
$$

where $\langle$,$\rangle is the intersection product on S$. On the other hand, by Riemann-Roch we have

$$
\begin{aligned}
\chi(L) & =\frac{1}{2}\left\langle[C],[C]+c_{1}(S)\right\rangle+\chi\left(\mathcal{O}_{S}\right) \\
& =\frac{1}{2}\left\langle[C],[C]-c_{1}(S)\right\rangle+\left\langle[C], c_{1}(S)\right\rangle+1 \\
& =g-1+\left\langle[C], c_{1}(S)\right\rangle+1 \\
& =g+\left\langle[C], c_{1}(S)\right\rangle .
\end{aligned}
$$

Furthermore, we have

$$
h^{2}(L)=h^{2}\left(S, \mathcal{O}_{S}(C)\right)=h^{0}\left(S, \omega_{S}(-C)\right) \leq h^{0}\left(S, \omega_{S}\right)=0,
$$

where the last equality holds since $S$ is rational. So, we see

$$
h^{0}(L) \geq \chi(L)=g+\left\langle[C], c_{1}(S)\right\rangle .
$$

To prove the vanishing of $h^{1}(L)$, we use the sequence

$$
H^{1}\left(S, \mathcal{O}_{S}\right) \rightarrow H^{1}\left(S, \mathcal{O}_{S}(C)\right) \rightarrow H^{1}\left(C, \mathcal{O}_{C}(C)\right) \rightarrow H^{2}\left(S, \mathcal{O}_{S}\right) .
$$

Since the higher cohomologies of $\mathcal{O}_{S}$ on $S$ vanish,

$$
h^{1}(S, L)=h^{1}\left(C, \mathcal{O}_{C}(C)\right) .
$$

By Serre duality and adjunction, we have

$$
h^{1}\left(C, \mathcal{O}_{C}(C)\right)=h^{0}\left(C, \omega_{C}(-C)\right)=h^{0}\left(C, \omega_{S}\right) .
$$

However, by the positivity hypothesis,

$$
\left\langle[C], c_{1}\left(\omega_{S}\right)\right\rangle<0,
$$

so $h^{0}\left(C, \omega_{S}\right)=0$.

Since the hypotheses of Lemma 2.3 hold, we may apply the conclusion: for $r=g-1+\int_{[C]} c_{1}(S)$ and pairwise distinct $p_{1}, \ldots, p_{r} \in C$, we have

$$
\epsilon_{*}\left(\prod_{i=1}^{r} \mathrm{ev}_{i}^{*}[\mathrm{pt}] \cap\left[\overline{\mathcal{M}}_{g, r}\left(S, c_{1}(L)\right)\right]^{\mathrm{vir}}\right)=\left[C, p_{1}, \ldots, p_{r}\right] \in A_{0}\left(\overline{\mathcal{M}}_{g, r}\right),
$$

where $[\mathrm{pt}] \in A_{0}(S, \mathbb{Z})$ is the class of (any) point as $S$ is rational.

Step 2. The rational surface $S$ can be deformed to a toric surface $\widehat{S}$ in a smooth family

$$
\mathcal{S} \rightarrow B
$$

over a rationally connected variety $B$ containing $S, \widehat{S}$ as special fibres. ${ }^{11}$ The line bundle $L$ can be deformed along with $S$ to a line bundle

$$
\widehat{L} \rightarrow \widehat{S}
$$

\footnotetext{
${ }^{11}$ There is no difficultly in finding such a deformation. The minimal model of $S$ is toric. The exceptional divisors can then be moved to toric fixed points.
} 
Since the virtual fundmental class is constructed in families [BF97],

$$
\epsilon_{*}\left(\prod_{i=1}^{r} \mathrm{ev}_{i}^{*}[\mathrm{pt}] \cap\left[\overline{\mathcal{M}}_{g, r}\left(S, c_{1}(L)\right)\right]^{\mathrm{vir}}\right)=\epsilon_{*}\left(\prod_{i=1}^{r} \mathrm{ev}_{i}^{*}[\mathrm{pt}] \cap\left[\overline{\mathcal{M}}_{g, r}\left(\widehat{S}, c_{1}(\widehat{L})\right)\right]^{\mathrm{vir}}\right) .
$$

We have therefore moved the calculation to the toric setting.

Step 3. The virtual localization formula of [GP99] applied to the toric surface $\widehat{S}$ immediately shows

$$
\epsilon_{*}\left(\prod_{i=1}^{n} \mathrm{ev}_{i}^{*}[\mathrm{pt}] \cap\left[\overline{\mathcal{M}}_{g, r}\left(\widehat{S}, c_{1}(\widehat{L})\right)\right]^{\mathrm{vir}}\right) \in R_{0}\left(\overline{\mathcal{M}}_{g, r}\right) .
$$

We have proven that the 0 -cycle $\left[C, p_{1}, \ldots, p_{r}\right] \in A_{0}\left(\overline{\mathcal{M}}_{g, r}\right)$ is tautological. If $0 \leq n \leq r$,

$$
\left[C, p_{1}, \ldots, p_{n}\right] \in A_{0}\left(\overline{\mathcal{M}}_{g, n}\right)
$$

must also be tautological (by applying the forgetful map).

\subsection{Variations}

Let $S$ be a nonsingular projective rational surface, and let

$$
C \subset S
$$

be a reduced, irreducible, nodal curve of arithmetic genus $g$ satisfying the positivity condition

$$
\int_{[C]} c_{1}(S)>0 \text {. }
$$

The statements and proofs of Lemma 2.3 and Theorem 1.4 are still valid for such curves ${ }^{12}$ : the 0 -cycle

$$
\left[C, p_{1}, \ldots, p_{n}\right] \in A_{0}\left(\overline{\mathcal{M}}_{g, n}\right)
$$

is tautological if $n \leq \operatorname{vdim} \overline{\mathcal{M}}_{g}(S,[C])$.

Can the positivity condition (3.3) be relaxed? Positivity was used in the proof of Theorem 1.4 only to prove that the associated linear series has the expected dimension. If $C \subset S$ is an irreducible nodal curve of arithmetic genus $g$ satisfying

$$
h^{1}\left(S, \mathcal{O}_{S}(C)\right)=0,
$$

then we can still conclude that the 0 -cycle

$$
\left[C, p_{1}, \ldots, p_{n}\right] \in A_{0}\left(\overline{\mathcal{M}}_{g, n}\right)
$$

is tautological if $n \leq \operatorname{vdim} \overline{\mathcal{M}}_{g}(S,[C])$.

According to the Harbourne-Hirschowitz conjecture [Har86, Hir89], the vanishing (3.4) should always hold if $S$ is sufficiently general. We therefore expect an affirmative answer to the following question.

Question 3.1. Let $C \subset S$ be an irreducible nonsingular (or an irreducible nodal) curve with no positivity assumption on $\int_{[C]} c_{1}(S)$. Is the 0 -cycle

$$
\left[C, p_{1}, \ldots, p_{n}\right] \in A_{0}\left(\overline{\mathcal{M}}_{g, n}\right)
$$

tautological for $n \leq \operatorname{vdim} \overline{\mathcal{M}}_{g}(S,[C])$ ?

\footnotetext{
${ }^{12}$ The points $p_{i}$ here are distinct and lie in the nonsingular locus of $C$.
} 
On the other hand, if $C \subset S$ is a reducible nodal curve, we obtain a parallel statement by applying the results above for each irreducible component separately. Here, each component $C_{v}$ with arithmetic genus $g_{v}$ must satisfy the positivity condition (3.3), and the number of markings plus the number of preimages of nodes must be bounded by the virtual dimension $\operatorname{vim} \overline{\mathcal{M}}_{g_{v}}\left(S,\left[C_{v}\right]\right)$.

\section{K3 surfaces}

\subsection{Beauville-Voisin classes}

On a nonsingular projective $K 3$ surface $S$, there exists a canonical zero cycle $c_{S} \in A_{0}(S, \mathbb{Z})$ of degree 1 satisfying the following three properties [BV04]:

- all points in $S$ lying on a (possibly singular) rational curve have class $c_{S} \in A_{0}(S, \mathbb{Z})$,

- the image of the intersection product $A_{1}(S, \mathbb{Z}) \otimes A_{1}(S, \mathbb{Z}) \rightarrow A_{0}(S, \mathbb{Z})$ lies in $\mathbb{Z} \cdot c_{S}$,

- the second Chern class $c_{2}(S)$ is equal to $24 c_{S}$.

The Beauville-Voisin subspace is defined by

$$
\mathrm{BV}=\mathbb{Z} \cdot c_{s} \subset A_{0}(S, \mathbb{Z}) .
$$

A point $p \in S$ is a Beauville-Voisin point if $[p] \in \mathrm{BV}$.

\subsection{Proof of Theorem 1.5}

The claim is trivial for genus $g=1$ since $\overline{\mathcal{M}}_{1,1}$ is rational. We can therefore assume $g \geq 2$. By Lemma 2.4, we have

$$
\epsilon_{*}\left(c^{*} H^{g-n} \cap \prod_{i=1}^{n} \operatorname{ev}_{i}^{*}\left[p_{i}\right] \cap\left[\overline{\mathcal{M}}_{g, n}(S,[C])\right]^{\mathrm{red}}\right)=\left[C, p_{1}, \ldots, p_{n}\right]
$$

in $A_{0}\left(\overline{\mathcal{M}}_{g, n}\right)$.

We briefly recall the notation used in (4.1). For $L=\mathcal{O}_{S}(C)$,

$$
c: \overline{\mathcal{M}}_{g, n}(S,[C]) \rightarrow \mathbb{P}\left(H^{0}(S, L)\right)
$$

is the map sending

$$
f:\left(\widehat{C}, \widehat{p}_{1}, \ldots, \widehat{p}_{n}\right) \rightarrow S
$$

to $f_{*}[\widehat{C}] \in|L|$ and $H$ is the hyperplane class of $|L|$. Since the points $p_{i}$ are all Beauville-Voisin, equality (2.4) immediately implies that the right hand side depends only upon the surface $S$ and the class

$$
\left[\mathcal{O}_{S}(C)\right] \in \operatorname{Pic}(S) \text {. }
$$

By Lemma 2.3 of [Huy16, Chapter 2], the line bundle $L$ is base point free and hence nef. Let

$$
L=L_{0}^{\otimes k}, \quad k \geq 1
$$

for $L_{0}$ primitive of degree

$$
d=2 g^{\prime}-2>0 .
$$

Then, $L_{0}$ is still nef, so $\left(S, L_{0}\right)$ is a quasi-polarized $K 3$ surface of degree $d$. Consider the moduli stack $\mathcal{F}_{d}$ of quasi-polarized $K 3$ surfaces $\left(\widehat{S}, \widehat{L}_{0}\right)$ of degree $d$. Let

$$
\pi: \mathcal{S} \rightarrow \mathcal{F}_{d}
$$

be the universal $K 3$ surface over $\mathcal{F}_{d}$ with universal polarization $\mathcal{L}_{0} \in A^{1}(\mathcal{S})$. The restriction of $\left(\mathcal{S}, \mathcal{L}_{0}\right)$ to the fibre over $\left(\widehat{S}, \widehat{L}_{0}\right) \in \mathcal{F}_{d}$ is isomorphic to $\left(\widehat{S}, \widehat{L}_{0}\right)$, see [PY20]. 
Consider furthermore the projective bundle

$$
\mathcal{P}=\mathbb{P}\left(R^{0} \pi_{*}\left(\left(\mathcal{L}_{0}\right)^{\otimes k}\right)\right) \rightarrow \mathcal{F}_{d}
$$

parametrizing elements in the linear system $\left(\mathcal{L}_{0}\right)^{\otimes k}$ on the fibres of $\mathcal{S}$. The projective bundle $\mathcal{P}$ is of fibre dimension $g$ by Theorem 1.8 of [Huy16, Chapter 2].

We can then obtain the left hand side of (2.4) as a fibre in a family of cycles parametrized by $\mathcal{F}_{d}$. Indeed, denote by $\mathcal{S}^{n}$ the $n$-fold self product of $\mathcal{S}$ over $\mathcal{F}_{d}$ and consider the following commutative diagram:

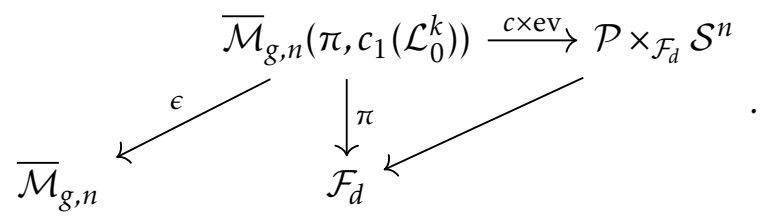

Here, $\overline{\mathcal{M}}_{g, n}\left(\pi, c_{1}\left(\mathcal{L}_{0}^{k}\right)\right)$ is the moduli space of stable maps to the fibres of $\pi$ of curve class equal to $c_{1}\left(\mathcal{L}_{0}^{k}\right)$ on the fibres of $\pi$. The map $c$ is the version of the previous map $c$ in families, and

$$
\mathrm{ev}=\left(\mathrm{ev}_{1}, \ldots, \mathrm{ev}_{n}\right)
$$

is the evaluation map corresponding to the $n$ points. Let

$$
\mathcal{H}=c_{1}\left(\mathcal{O}_{\mathcal{P}}(1)\right)
$$

be the hyperplane class of the projective bundle $\mathcal{P}$, and let

$$
c_{\mathcal{S}}=\frac{1}{24} c_{2}\left(\Omega_{\pi}\right) \in A^{2}(\mathcal{S})
$$

be the relative Beauville-Voisin class of the family

$$
\pi: \mathcal{S} \rightarrow \mathcal{F}_{d} .
$$

Consider the cycle $\mathcal{Z} \in A^{3 g-3+n}\left(\overline{\mathcal{M}}_{g, n} \times \mathcal{F}_{d}\right)$ defined by

$$
\mathcal{Z}=(\epsilon, \pi)_{*}\left(c^{*} \mathcal{H}^{g-n} \cap \prod_{i=1}^{n} \operatorname{ev}_{i}^{*} c_{\mathcal{S}} \cap\left[\overline{\mathcal{M}}_{g, n}\left(\pi, c_{1}\left(\mathcal{L}_{0}^{k}\right)\right]^{\mathrm{red}}\right) .\right.
$$

The fibre of $\mathcal{Z}$ over $\left(S, L_{0}\right)$ is equal to the left hand side of (2.4).

By Proposition 2.1, we need only show that the fibre of $\mathcal{Z}$ over the general point of $\mathcal{F}_{d}$ is tautological. So let

$$
\left(\widehat{S}, \widehat{L}_{0}\right) \in \mathcal{F}_{d}
$$

be a general quasi-polarized $K 3$ of degree $d$. By the existence result of [Che99], the linear system $\left|\widehat{L}_{0}^{\otimes k}\right|$ contains an irreducible nodal rational curve

$$
R \subset \widehat{S}
$$

Furthermore, since $(\widehat{S}, \widehat{L})$ is general, we can assume that $\widehat{L}_{0}$ and thus $\widehat{L}_{0}^{\otimes k}$ are basepoint free (see Theorem 4.2 of [Huyl6, Chapter 2]). By Bertini's theorem, the general member $\widehat{C}$ of the linear system $\left|L_{0}^{\otimes k}\right|$ intersects the rational curve $R$ only in reduced points. The number of these intersection points is exactly

$$
\langle[\widehat{C}],[\widehat{C}]\rangle=2 g-2,
$$

which is at least $g$ (since we assume $g \geq 2$ ). Choose distinct points

$$
q_{1}, \ldots, q_{n} \in R \cap \widehat{C} \text {. }
$$

Certainly all the $q_{i}$ are Beauville-Voisin points since they lie on the rational curve $R$. Since

$$
R, \widehat{C} \in\left|\widehat{L}_{0}^{k}\right| \text {, }
$$


there exists a pencil of curves connecting $\left(\widehat{C}, q_{1}, \ldots, q_{n}\right)$ and $\left(R, q_{1}, \ldots, q_{n}\right)$. The 0 -cycle given by $\left[\left(R, q_{1}, \ldots, q_{n}\right)\right] \in A^{0}\left(\overline{\mathcal{M}}_{g, n}\right)$ is clearly tautological, since the point lies in the image of

$$
\overline{\mathcal{M}}_{0, n+2 g} \rightarrow \overline{\mathcal{M}}_{g, n} .
$$

Therefore, $\left[\left(\widehat{C}, q_{1}, \ldots, q_{n}\right)\right]$ is tautological.

We isolate part of the above proof as a separate corollary for later application.

Corollary 4.1. Let $S$ be a $K 3$ surface with $L \in \operatorname{Pic}(S)$. There exists a $\mathbb{Q}$-linear map

$$
\Phi: A_{0}\left(S^{n}\right) \rightarrow A_{0}\left(\overline{\mathcal{M}}_{g, n}\right)
$$

defined by

$$
\Phi(\alpha)=\epsilon_{*}\left(c^{*} H^{g-n} \cap \operatorname{ev}^{*} \alpha \cap\left[\overline{\mathcal{M}}_{g, n}\left(S, c_{1}(L)\right)\right]^{\mathrm{red}}\right) .
$$

For an irreducible nonsingular projective curve $C \subset S$ of genus $g \geq 1$ in the linear series $L$ and distinct points $p_{1}, \ldots, p_{n} \in C$ we have

$$
\left[\left(C, p_{1}, \ldots, p_{n}\right)\right]=\Phi\left(\left[\left(p_{1}, \ldots, p_{n}\right)\right]\right) .
$$

Moreover, $\Phi\left(\left(c_{S}\right)^{\times n}\right)$ is tautological.

\subsection{Quotients} let

The symmetric group $S_{n}$ acts on $\overline{\mathcal{M}}_{g, n}$ by permuting the markings. For a partition $\mu=\left(n_{1}, \ldots, n_{\ell}\right)$ of $n$,

$$
S_{\mu}=S_{n_{1}} \times \cdots \times S_{n_{\ell}} \subset S_{n}
$$

be the subgroup permuting elements within the blocks defined by $\mu$. The stack quotient

$$
\overline{\mathcal{M}}_{g, \mu}=\overline{\mathcal{M}}_{g, n} / S_{\mu}
$$

parametrizes curves

$$
\left(C,\left(\left\{p_{i, 1}, \ldots, p_{i, n_{i}}\right\}\right)_{i=1, \ldots, \ell}\right)
$$

together with $\ell$ pairwise disjoint sets of marked points with sizes $n_{i}$ according to the partition $\mu$. The quotient map

$$
\pi: \overline{\mathcal{M}}_{g, n} \rightarrow \overline{\mathcal{M}}_{g, \mu}
$$

allows us to define the tautological ring $R^{*}\left(\overline{\mathcal{M}}_{g, \mu}\right)$ as the image of $R^{*}\left(\overline{\mathcal{M}}_{g, n}\right)$ via push-forward by $\pi$. The composition

$$
\pi_{*} \pi^{*}: A^{*}\left(\overline{\mathcal{M}}_{g, \mu}\right) \rightarrow A^{*}\left(\overline{\mathcal{M}}_{g, \mu}\right)
$$

is given by multiplication by $\left|S_{\mu}\right|$. Therefore, to check if a cycle $\alpha$ on $\overline{\mathcal{M}}_{g, \mu}$ is tautological, it suffices to check that $\pi^{*}(\alpha)$ is tautological on $\overline{\mathcal{M}}_{g, n}$.

The following result for the quotient moduli spaces $\overline{\mathcal{M}}_{g, \mu}$ is parallel to Theorem 1.5 for $\overline{\mathcal{M}}_{g, n}$.

Theorem 4.2. Let $C \subset S$ be an irreducible nonsingular curve of genus $g$ on a $K 3$ surface. Let $0 \leq n \leq g$ and fix a partition $\mu=\left(n_{1}, \ldots, n_{\ell}\right)$ of $n$. Let

$$
\left(p_{i, j}\right)_{\substack{i=1, \ldots, \ell \\ j=1, \ldots, n_{i}}}
$$

be a collection of distinct points $p_{i, j} \in C$ satisfying

$$
\left[p_{i, 1}\right]+\left[p_{i, 2}\right]+\cdots+\left[p_{i, n_{i}}\right] \in \mathrm{BV}
$$

for all $1 \leq i \leq \ell$. Then, the 0 -cycle

$$
\left[C,\left(\left\{p_{i, 1}, \ldots, p_{i, n_{i}}\right\}\right)_{i=1, \ldots, \ell}\right] \in A_{0}\left(\overline{\mathcal{M}}_{g, \mu}\right)
$$

is tautological. 
Proof. It suffices to show that the pullback $\pi^{*}\left(\left[C,\left(p_{i, j}\right)_{i}\right]\right)$ is tautological. Fix an ordering $\mathbf{p}=\left(p_{i, j}\right)_{i}$ of all the markings. The pullback is exactly given by

$$
\pi^{*}\left(\left[C,\left(p_{i, j}\right)_{i}\right]\right)=\sum_{\sigma \in S_{\mu}}[\sigma(C, \mathbf{p})] .
$$

Using Corollary 4.1, we can write the result as $\Phi(\Sigma(\mathbf{p}))$ for the sum

$$
\Sigma(\mathbf{p})=\sum_{\sigma \in S_{\mu}}[\sigma \cdot \mathbf{p}] \in A_{0}\left(S^{n}\right),
$$

where we have used the natural permutation action of $S_{n}$ on $S^{n}$.

We claim that the cycle $\Sigma(\mathbf{p})$ only depends on the blockwise sums

$$
\Sigma_{i}(\mathbf{p})=\sum_{j}\left[p_{i, j}\right] \in A_{0}(S)
$$

for $i=1, \ldots, \ell$. Blockwise dependence together with the hypothesis

$$
\Sigma_{i}(\mathbf{p}) \in \mathrm{BV}
$$

immediately yields the result of Theorem 4.2 (since we can exchange all the $p_{i, j}$ for Beauville-Voisin points).

It remains only to prove the blockwise dependence. We first observe that we can write $\Sigma(\mathbf{p})$ as a product

$$
\Sigma(\mathbf{p})=\sum_{\sigma \in S_{\mu}}[\sigma \cdot \mathbf{p}]=\prod_{i=1}^{\ell} \sum_{\sigma_{i} \in S_{n_{i}}}\left[\sigma_{i} \cdot\left(p_{i, j}\right)_{j=1, \ldots, n_{i}}\right],
$$

where we recall that $S_{\mu}$ is the product of the groups $S_{n_{i}}$. It suffices then to show that the $i$ th factor in the above product only depends on the sum $\Sigma_{i}(\mathbf{p})$. The latter claim amounts to a reduction to the case of the partition $\mu=(n)$ where all the markings are permuted.

Let $P=\left\{p_{1}, \ldots, p_{n}\right\}$. We will write

$$
\Sigma(\mathbf{p})=\sum_{\sigma \in S_{n}}\left[\left(p_{\sigma(1)}, \ldots, p_{\sigma(n)}\right)\right]
$$

as a sum of terms depending only upon

$$
\theta=\Sigma_{1}(\mathbf{p})=\left[p_{1}\right]+\cdots+\left[p_{n}\right]
$$

using a simple inclusion-exclusion strategy.

We illustrate the strategy in the case of $n=3$. We start with the formula

$$
\theta^{\times 3}=\sum_{q_{1}, q_{2}, q_{3} \in P}\left[\left(q_{1}, q_{2}, q_{3}\right)\right] .
$$

To obtain $\Sigma(\mathbf{p})$, we must substract all summands where there is a pair $i \neq j$ with $q_{i}=q_{j}$. Let

$$
\Delta_{12,3}, \Delta_{13,2}, \Delta_{23,1}: S^{2} \rightarrow S^{3}
$$

be the three diagonal maps. The cycle

$$
\theta^{\times 3}-\left(\Delta_{12,3}\right)_{*}\left(\theta^{\times 2}\right)-\left(\Delta_{13,2}\right)_{*}\left(\theta^{\times 2}\right)-\left(\Delta_{23,1}\right)_{*}\left(\theta^{\times 2}\right)
$$

is equal to $\Sigma(\mathbf{p})$ minus 2 times the cycle

$$
\left[\left(p_{1}, p_{1}, p_{1}\right)\right]+\left[\left(p_{2}, p_{2}, p_{2}\right)\right]+\left[\left(p_{3}, p_{3}, p_{3}\right)\right] .
$$

We can cancel the error term by adding a correction $2\left(\Delta_{123}\right)_{*}(\theta)$ by the small diagonal:

$$
\Sigma(\mathbf{p})=\theta^{\times 3}-\left(\Delta_{12,3}\right)_{*}\left(\theta^{\times 2}\right)-\left(\Delta_{13,2}\right)_{*}\left(\theta^{\times 2}\right)-\left(\Delta_{23,1}\right)_{*}\left(\theta^{\times 2}\right)+2\left(\Delta_{123}\right)_{*}(\theta) .
$$

Such an inclusion-exclusion strategy is valid for all $n \geq 1$. 


\section{Other surface geometries}

\subsection{Enriques surfaces}

An Enriques surface $E$ is a free $\mathbb{Z}_{2}$ quotient of a nonsingular projective $K 3$ surface $S$ :

$$
E=S / \mathbb{Z}_{2} .
$$

Conjecture 5.1. The moduli point of an irreducible nonsingular curve $C \subset E$ of genus $g \geq 2$ determines a tautological 0 -cycle in $\overline{\mathcal{M}}_{g}$.

There is a clear strategy for the proof of Conjecture 5.1. The curve $C$ is expected to move in a linear series $|L|$ on $E$ of dimension $g-1$. We therefore expect to find irreducible curves $\widehat{C} \in|L|$ with $g-1$ nodes. The issue can be formulated as the nonemptiness of certain Severi varieties for linear systems on Enriques surfaces which is currently being studied, see [CDGK20]. Once it is shown that the linear series $|L|$ contains an irreducible $(g-1)$-nodal curve $\widehat{C} \subset E$, the final step is to prove that the 0 -cycle

$$
[\widehat{C}] \in A_{0}\left(\overline{\mathcal{M}}_{g}\right)
$$

is always tautological. In fact, the following stronger result holds.

Proposition 5.2. The locus of irreducible $(g-1)$-nodal curves in $\overline{\mathcal{M}}_{g, 1}$ is rational. In particular, every such curve defines a tautological cycle

$$
[\widehat{C}, p] \in R_{0}\left(\overline{\mathcal{M}}_{g, 1}\right) .
$$

Proof. The closure of the locus of $(g-1)$-nodal curves is parametrized by the gluing map

$$
\xi: \overline{\mathcal{M}}_{1,1+2(g-1)} \rightarrow \overline{\mathcal{M}}_{g}
$$

taking a curve $\left(X, p, q_{1}, q_{1}^{\prime}, \ldots, q_{g-1}, q_{g-1}^{\prime}\right)$ of genus 1 with $1+2(g-1)$ markings and identifying the $g-1$ pairs $q_{j}, q_{j}^{\prime}$ of points. The group

$$
G=(\mathbb{Z} / 2 \mathbb{Z})^{g-1} \rtimes S_{g-1}
$$

acts on $\overline{\mathcal{M}}_{1,1+2(g-1)}$ : the $j$ th factor $\mathbb{Z} / 2 \mathbb{Z}$ switches the two points $q_{j}, q_{j}^{\prime}$ and the group $S_{g-1}$ permutes the $n$ pairs of points among each other. Since the gluing map $\xi$ is invariant under this action, it factors through the map

$$
\tilde{\xi}: \overline{\mathcal{M}}_{1,1+2(g-1)} / G \rightarrow \overline{\mathcal{M}}_{g, 1},
$$

which is birational onto its image.

To prove $\mathcal{M}=\mathcal{M}_{1,1+2(g-1)} / G$ is rational, we take a modular reinterpretation. Instead of remembering the $2(g-1)$ points $q_{j}, q_{j}^{\prime}$ on $X$ individually, we only remember the set

$$
\left\{D_{j}=q_{j}+q_{j}^{\prime}: j=1, \ldots, g-1\right\}
$$

of $g-1$ effective divisors of degree 2 on the curve $X$. We therefore have a birational identification

$$
\mathcal{M} \leftrightarrow\left\{\left(X, p,\left(D_{j}\right)_{j=1}^{g-1}\right): \begin{array}{c}
X \text { nonsingular elliptic curve with origin } p, \\
D_{j} \subset X \text { effective degree } 2 \text { divisors }
\end{array}\right\} / S_{g-1},
$$

where $S_{g-1}$ acts by permuting the divisors $D_{1}, \ldots, D_{g-1}$.

An effective divisor $D_{j} \subset X$ is equivalent to the data of the degree 2 line bundle

$$
\mathcal{L}_{j}=\mathcal{O}\left(D_{j}\right)
$$

together with an element

$$
s_{j} \in \mathbb{P}\left(H^{0}\left(X, \mathcal{L}_{j}\right)\right) \cong \mathbb{P}^{1} .
$$


Furthermore, the class of the line bundle $\mathcal{L}_{j}$ is equivalent to specifying a point $l_{j} \in X$, by the correspondence sending $l_{j}$ to $\mathcal{O}\left(p+l_{j}\right)$, where $p \in X$ is the origin. We define

$$
\mathcal{P}=\left\{\left(X, p,\left(l_{j}\right)_{j=1}^{g-1},\left(s_{j}\right)_{j=1}^{g-1}\right): \quad \begin{array}{c}
X \text { nonsingular elliptic curve with origin } p, \\
l_{j} \in E \\
s_{j} \in \mathbb{P}\left(H^{0}\left(X, \mathcal{O}\left(p+l_{j}\right)\right)\right)
\end{array}\right\} .
$$

We have a birational identification

$$
\mathcal{M} \longleftrightarrow \mathcal{P} / S_{g-1}
$$

By forgetting the projective sections $s_{j}$, we obtain a map

$$
\mathcal{P} \rightarrow \mathcal{S}
$$

to the space $\mathcal{S}$ parametrizing tuples $\left(X, p,\left(l_{j}\right)_{j}\right)$ as above. The above forgetful map is a $\left(\mathbb{P}^{1}\right)^{g-1}$-bundle which descends (birationally) to a $\left(\mathbb{P}^{1}\right)^{g-1}$-bundle

$$
\mathcal{P} / S_{g-1} \rightarrow \mathcal{S} / S_{g-1}
$$

on the quotient. The base, the moduli space parameterizing the data

$$
\left(X, p,\left(l_{j}\right)_{j}\right)
$$

up to permutations of the $l_{j}$ by $S_{g-1}$, is easily seen to be rational using, to start, the rationality of the universal family of $\mathrm{Jac}_{2}$ over $\overline{\mathcal{M}}_{1,1}$.

Using the rationality of $\overline{\mathcal{M}}_{1,10}$, Proposition 5.2 can be easily strengthened to show that the locus of irreducible $(g-1)$-nodal curves in $\overline{\mathcal{M}}_{g, 9}$ is rational. In particular, every such curve defines a tautological cycle

$$
\left[\widehat{C}, p_{1}, \ldots, p_{9}\right] \in R_{0}\left(\overline{\mathcal{M}}_{g, 9}\right) .
$$

\subsection{Abelian surfaces}

Let $A$ be a nonsingular projective Abelian surface. An irreducible nonsingular curve

$$
C \subset A
$$

is expected to move in a linear series $|L|$ of dimension $g-2$. We therefore expect to find curves $\widehat{C} \in|L|$ with $g-2$ nodes. Unfortunately the strategy that we have outlined in the case of Enriques surfaces fails here! The locus of irreducible $(g-2)$-nodal curves in $\overline{\mathcal{M}}_{g}$ is not always rational. The irrationality of the locus of 7 nodal curves in $\overline{\mathcal{M}}_{9}$ was proven with Faber using the non-triviality (and representation properties) of $H^{14,0}\left(\overline{\mathcal{M}}_{2,14}\right)$. A study of the Kodaira dimensions of the loci of curves with multiple nodes in many (other) cases can be found in [Sch18].

Nevertheless, an affirmative answer to the following question appears likely.

Question 5.3. Does every irreducible nonsingular curve $C \subset A$ of genus $g$ determine a tautological 0 -cycle $[C] \in A_{0}\left(\overline{\mathcal{M}}_{g}\right)$ ?

Another approach to Question 5.3 is to use curves on $K 3$ surfaces via the Kummer construction. Using the involution

$$
\iota: A \rightarrow A, \quad a \mapsto-a,
$$

we obtain a $K 3$ surface $S$ by resolving the singular points of the quotient $A / \iota$. If $C$ does not meet any of these 16 points (which are the fixed-points of $\iota$ ), the corresponding rational map

$$
A \rightarrow A /\llcorner\rightarrow S
$$


is defined around $C \subset A$ and sends $C$ to a curve $C^{\prime} \subset S$. The map $C \rightarrow C^{\prime}$ is either a double cover (in which case it must be étale with $C^{\prime}$ smooth) or birational. In the first case, $\left[C^{\prime}\right]$ is tautological by Theorem 1.5 which may help in proving that $[C]$ is tautological. In the second case, the curve $C$ is the normalization of $C^{\prime}$, and we would require a variant of Theorem 1.5 to show that, under suitable conditions, the normalization of an irreducible, nodal curve in a $K 3$ surface is tautological.

\subsection{Surfaces of general type}

Let $S$ bs a nonsingular projective surface of general type. A curve $C \subset S$ is canonical if

$$
\omega_{S} \stackrel{\sim}{=} \mathcal{O}_{S}(C) \text {. }
$$

The most basic question which can be asked is the following.

Question 5.4. Does every irreducible nonsingular canonical curve $C \subset S$ of genus $g$ determine a tautological 0 -cycle $[C] \in A_{0}\left(\overline{\mathcal{M}}_{g}\right)$ ?

For surfaces $S$ arising as complete intersections in projective space, the answer to Question 5.4 is yes (since complete intersection curves are easily seen to determine tautological 0 -cycles by degenerating their defining equations to products of linear factors). However, even for surfaces of general type arising as double covers of $\mathbb{P}^{2}$, the issue does not appear trivial (even though the canonical curves there are realized as concrete double covers of plane curves). In fact, Question 5.4 is completely open in almost all cases.

\section{Cyclic covers}

If a nonsingular projective complex curve $C$ admits a Hurwitz covering of $\mathbb{P}^{1}$ ramified over only 3 points of $\mathbb{P}^{1}$, then $C$ can be defined over $\overline{\mathbb{Q}}$ by Belyi's Theorem. Speculation 1.3 , for $n=0$, then suggests that the moduli point of $C$ is tautological. The following result proves a special case for cyclic covers. ${ }^{13}$

Theorem 6.1. Let $C$ be a nonsingular projective curve of genus $g$ admitting a cyclic cover

$$
\varphi: C \rightarrow \mathbb{P}^{1}
$$

ramified over exactly three points of $\mathbb{P}^{1}$ and with total ramification over at least one of them. Let $p_{1}, \ldots, p_{n} \in C$ be the ramification points of $\varphi$ (in some order). Then, the 0 -cycle

$$
\left[C, p_{1}, \ldots, p_{n}\right] \in A_{0}\left(\overline{\mathcal{M}}_{g, n}\right)
$$

is tautological.

Proof. The basic idea is that a cyclic cover of $\mathbb{P}^{1}$ can (essentially) be cut out by a single equation in a projective bundle over $\mathbb{P}^{1}$. Indeed, after a change of coordinates, we can assume that the branch points of $\varphi$ are given by

$$
0,1,2 \in \mathbb{P}^{1} \text {. }
$$

Let $k$ be the degree of $\varphi$, and let $a, b, c \in \mathbb{Z} / k \mathbb{Z}$ be the monodromies of $\varphi$ at the branch points $0,1,2$ satisfying

$$
a+b+c=0 \in \mathbb{Z} / k \mathbb{Z} .
$$

Assume that the total ramification occurs over 0 . Then $a$ is coprime to $k$, and, by applying an automorphism of $\mathbb{Z} / k \mathbb{Z}$, we may assume $a=1$. We can then choose representatives

$$
b, c \in\{1, \ldots, k-1\}
$$

\footnotetext{
${ }^{13}$ Following the notation of [Sv18], Theorem 6.1 shows that the 0 -cycle

$$
\left[\overline{\mathcal{H}}_{g, \mathbb{Z} / k \mathbb{Z},(a, b, c)}\right] \in A_{0}\left(\overline{\mathcal{M}}_{g, n}\right)
$$
}

is tautological for $a, b, c \in \mathbb{Z} / k \mathbb{Z}$ where at least one of $a, b, c$ is coprime to $k$. 
such that $a+b+c=k$.

With these choices in place, we see that (birationally) the curve $C$ is cut out in the projectivization of the line bundle $\mathcal{O}_{\mathbb{P}^{1}}(1)$ over $\mathbb{P}^{1}$ by the equation

$$
y^{k}=x \cdot(x-1)^{b} \cdot(x-2)^{c},
$$

where $x$ is a coordinate on the base $\mathbb{P}^{1}$. We view the right hand side of (6.1) as a section of

$$
\mathcal{O}_{\mathbb{P}^{1}}(1+b+c)=\mathcal{O}_{\mathbb{P}^{1}}(k)
$$

where $y$ is the coordinate on (the total space of) the line bundle $\mathcal{O}_{\mathbb{P}^{1}}(1)$ over $\mathbb{P}^{1}$.

We say that $C$ is cut out birationally since, for $b, c \neq 1$, the above curve will have singularities at

$$
(x, y)=(1,0),(2,0) \text {. }
$$

The singularities can be resolved by performing a specific sequence of iterated blowups (as will be explained in the next paragraph). After finitely many steps, we will obtain $C$ sitting inside a blowup $S$ of

$$
\mathbb{P}=\mathbb{P}\left(\mathcal{O}_{\mathbb{P}^{1}}(1) \oplus \mathcal{O}_{\mathbb{P}^{1}}\right),
$$

which is a nonsingular rational surface. In order to conclude by applying Theorem 1.4, we will have to check that

$$
\int_{[C]} c_{1}(S)>0
$$

holds and that the number $n$ of ramification points of $\varphi$ is at most equal to vdim $\overline{\mathcal{M}}_{g}(S,[C])$.

The original curve $C_{0}$ in $\mathbb{P}$ is easily seen to be of class $\beta=k c_{1}\left(\mathcal{O}_{\mathbb{P}}(1)\right)$, and we have

$$
\int_{\beta} c_{1}(\mathbb{P})=3 k
$$

If $b>1$, then $C_{0}$ has a singularity of multiplicity $b$ at $(x, y)=(1,0)$. For the coordinate $z=y /(x-1)$ on the blowup of $\mathbb{P}$ at $(1,0)$, the strict transform of $C_{0}$ is locally cut out by $z^{k-b}=(x-1)^{b}$. The relevant intersection number

$$
\int_{\beta-b E_{1}} c_{1}\left(\mathrm{Bl}_{(1,0)} \mathbb{P}\right)=3 k-b
$$

has exactly decreased by the multiplicity $b$ of $C_{0}$ at $(1,0)$.

We can continue the process of blowing-up the singular point and taking the strict transform. After $j$ steps, the curve still has a local equation of the form $z_{1}^{e_{j}}=z_{2}^{f_{j}}$. We started with $\left(e_{0}, f_{0}\right)=(k, b)$ and obtained

$$
\left(e_{1}, f_{1}\right)=(k-b, b)
$$

in the first step. In general, the pairs $\left(e_{j}, f_{j}\right)$ are then obtained by performing a Euclidean algorithm starting from $(k, b)$. The multiplicity of the singular point after the $j$ th step is exactly $\min \left(e_{j}, f_{j}\right)$. The process terminates after finitely many steps (when the minimum of $e_{j}, f_{j}$ is either 0 or 1 ). Then, the local equation is $z^{g}=1$ or $z^{g}=z^{\prime}$, which is nonsingular.

Denote by $\mathrm{ms}(e, f)$ the sum of the multiplicities of the singular points that occur in the desingularization of $z_{1}^{e}=z_{2}^{f}$ in the above manner. The function is uniquely determined by the axioms

$$
\begin{aligned}
& \text { - } \operatorname{ms}(e, f)=\operatorname{ms}(f, e), \\
& \text { - } \operatorname{ms}(e, 0)=\operatorname{ms}(e, 1)=0, \\
& \text { - } \operatorname{ms}(e, f)=f+\operatorname{ms}(e-f, f), \text { for } e \geq f \text {. }
\end{aligned}
$$

By the above analysis, the curve $C \subset S$ obtained by desingularizing $C_{0} \subset \mathbb{P}$ satisfies

$$
\int_{[C]} c_{1}(S)=\int_{\beta} c_{1}(\mathbb{P})-\mathrm{ms}(k, b)-\mathrm{ms}(k, c)=3 k-\mathrm{ms}(k, b)-\mathrm{ms}(k, c) .
$$


In order to show positivity, we must bound $\operatorname{ms}(e, f)$ from above. By induction, for $(e, f) \neq(1,1)$, we obtain:

$$
\operatorname{ms}(e, f) \leq e+f-R(e, f), \text { with } R(e, f)= \begin{cases}\operatorname{gcd}(e, f) & \text { if } \operatorname{gcd}(e, f)>1 \\ 3 & \text { otherwise }\end{cases}
$$

Then, we have

$$
\int_{[C]} c_{1}(S) \geq 3 k-k-b-k-c+R(k, b)+R(k, c)=1+R(k, b)+R(k, c) \geq 1 .
$$

For the virtual dimension we obtain

$$
\operatorname{vdim} \overline{\mathcal{M}}_{g}(S,[C])=\int_{[C]} c_{1}(S)+g-1 \geq g+R(k, b)+R(k, c) .
$$

On the other hand, the number of ramification points equals

$$
n=1+\operatorname{gcd}(k, b)+\operatorname{gcd}(k, c)
$$

so we have

$$
\operatorname{vdim}-n \geq g-1+\underbrace{(R(k, b)-\operatorname{gcd}(k, b))}_{\geq 0}+\underbrace{(R(k, c)-\operatorname{gcd}(k, c))}_{\geq 0} \geq g-1,
$$

which we can assume to be nonnegative. We have thus verified the assumptions of Theorem 1.4.

Without the assumption of total ramification over one of the three points, the proof technique above no longer works. Indeed, for $k=30$ and

$$
(a, b, c)=(2,3,25),
$$

a desingularization procedure over $x=0,1,2$ as in the above proof would result in a curve $C$ in $S$ satisfying

$$
\int_{[C]} c_{1}(S)=-20
$$

which cannot be remedied by applying an automorphism of $\mathbb{Z} / 30 \mathbb{Z}$. Nevertheless, we expect Theorem 6.1 to hold without the assumption of total ramification and even without the assumption of the cover being cyclic.

\section{Summing to tautological cycles}

\subsection{Existence}

As the examples $\overline{\mathcal{M}}_{1, n \geq 11}$ show, the Chow group of 0 -cycles on $\overline{\mathcal{M}}_{g, n}$ can be infinite dimensional over $\mathbb{Q}$. The general point of $\overline{\mathcal{M}}_{g, n}$ may not determine a tautological 0 -cycle. However, by adding points (with the number of points uniformly bounded in terms of $g, n$ ), we can arrive at a tautological 0-cycle. For technical reasons, we formulate the result for the coarse moduli space $\bar{M}_{g, n}$.

Proposition 7.1. Given $g, n$ with $2 g-2+n>0$, there exists an integer $T=T(g, n) \geq 1$ satisfying the following property: for any point

$$
Q_{1}=\left(C, p_{1}, \ldots, p_{n}\right) \in \bar{M}_{g, n},
$$

we can find $Q_{2}, \ldots, Q_{T} \in \bar{M}_{g, n}$ such that

$$
\left[Q_{1}\right]+\ldots+\left[Q_{T}\right] \in A_{0}\left(\bar{M}_{g, n}\right)
$$

is tautological. 
Proof (suggested by A. Kresch). By standard arguments using the results of Section 2, we may take $Q=Q_{1}$ to be a general point of $\bar{M}_{g, n}$. We then choose a very ample divisor class

$$
H \in A^{1}\left(\bar{M}_{g, n}\right) .
$$

Since $Q$ is a nonsingular point of $\bar{M}_{g, n}$, general hyperplane sections

$$
H_{1}, \ldots, H_{3 g-3+n} \in|H|
$$

through $Q$ will intersect transversely in a union of reduced points

$$
\alpha=\left[Q_{1}\right]+\cdots+\left[Q_{T}\right],
$$

with $T=\operatorname{deg}\left(\bar{M}_{g, n}, H\right)$. On the other hand, since all divisor classes on $\bar{M}_{g, n}$ are tautological, the class $\alpha$ is also tautological.

Remark 7.2. Since the push-forward along the basic map

$$
\overline{\mathcal{M}}_{g, n} \rightarrow \bar{M}_{g, n}
$$

is an isomorphism of $\mathbb{Q}$-Chow groups, we can derive a version of Proposition 7.1 with $\bar{M}_{g, n}$ replaced by $\overline{\mathcal{M}}_{g, n}$. However, $T(g, n)$ for $\bar{M}_{g, n}$, may differ from the corresponding number for $\overline{\mathcal{M}}_{g, n}:$ if $Q_{i} \in \overline{\mathcal{M}}_{g, n}$ has nontrivial automorphisms, then the cycle $\left[Q_{i}\right] \in A_{0}\left(\bar{M}_{g, n}\right)$ corresponds to the cycle

$$
\left|\operatorname{Aut}\left(Q_{i}\right)\right| \cdot\left[Q_{i}\right] \in A_{0}\left(\overline{\mathcal{M}}_{g, n}\right) .
$$

\subsection{Minimality}

We denote by $T(g, n)$ the minimal integer having the property described in Proposition 7.1. The proof of Proposition 7.1 used the degree of $\overline{\mathcal{M}}_{g, n}$, but there are several other geometric approaches to bounding $T(g, n)$. For example, we could use instead the Hurwitz cycle results of [FP05]. After fixing a degree $d \geq 1$, points $q_{1}, \ldots, q_{b} \in \mathbb{P}^{1}$, and partitions $\lambda_{1}, \ldots, \lambda_{b}$ of $d$, the sum of all points $\left[\left(C,\left(p_{i}\right)_{i}\right)\right]$ satisfying

- there exists a degree $d$ map $C \rightarrow \mathbb{P}^{1}$ with ramification profile $\lambda_{j}$ over $q_{j} \in \mathbb{P}^{1}$,

- with $\left(p_{i}\right)_{i}$ the set of preimages of the points $q_{1}, \ldots, q_{b}$

is tautological by [FP05]. Since every genus $g$ curve $C$ admits some map $C \rightarrow \mathbb{P}^{1}$, the result above implies that adding to $[C] \in A_{0}\left(\overline{\mathcal{M}}_{g}\right)$ all cycles $\left[C^{\prime}\right]$ for curves $C^{\prime} \rightarrow \mathbb{P}^{1}$ with the same branch points and ramification profiles as $C \rightarrow \mathbb{P}^{1}$ gives a tautological class. Hence, we bound $T(g, 0)$ in terms of a suitable Hurwitz number. A similar strategy works for any $n$ by including the markings $p_{1}, \ldots, p_{n} \in C$ among the ramification data of $C \rightarrow \mathbb{P}^{1}$.

However, these approaches will likely not yield optimal bounds. In all the cases listed in Figure 1, the space $\overline{\mathcal{M}}_{g, n}$ is rationally connected, so

$$
T(g, n)=1
$$

which is far below the bounds.

A different perspective on the question is to study the behavior of $T(g, n)$ for fixed $g$ as $n \rightarrow \infty$. The following result shows that the asymptotic growth in $n$ is at most linear.

Proposition 7.3. Let $(g, n)$ satisfy $2 g-2+n>0$. Then,

$$
T(g, n+m) \leq(g m+1) \cdot T(g, n)
$$

for all $m \geq 0$. 
Proof. The natural forgetful map

$$
v: \overline{\mathcal{M}}_{g, n+m} \rightarrow \overline{\mathcal{M}}_{g, n}
$$

has a section $\sigma$ defined by the following construction: $\sigma\left(\left(C, p_{1}, \ldots, p_{n}\right)\right)$ is the curve obtained by gluing a chain of rational curves containing the markings $p_{n}, \ldots, p_{n+m}$ at the previous position of $p_{n} \in C$.

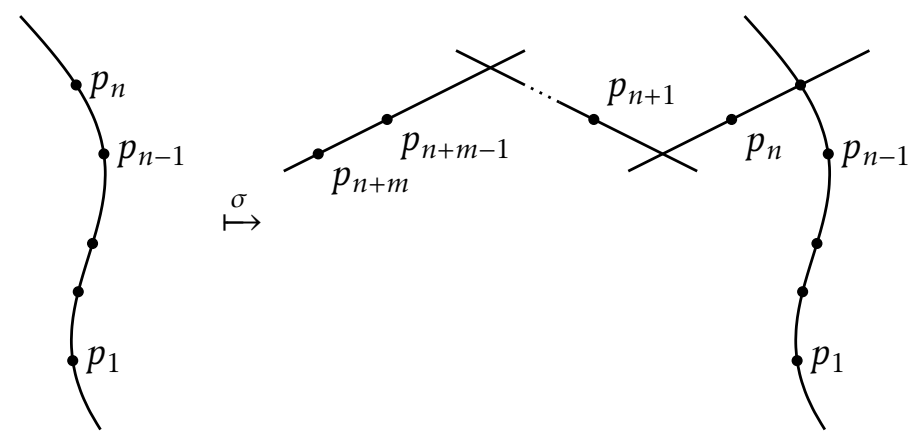

The section $\sigma$ is a composition of suitable boundary gluing maps, so the push-forward of a tautological cycle via $\sigma$ is tautological.

Let $Q \in \mathcal{M}_{g, n}$ be a moduli point with a nonsingular domain curve $C$. We claim: for every $Q_{1} \in v^{-1}(Q)$, there exist $Q_{2}, \ldots, Q_{g m+1} \in v^{-1}(Q)$ satisfying

$$
\left[Q_{1}\right]+\cdots+\left[Q_{g m+1}\right]=(g m+1)[\sigma(Q)] \in A_{0}\left(v^{-1}(Q)\right) .
$$

Assuming the above claim, we can easily finish the proof.

Let $Q_{1} \in \mathcal{M}_{g, n+m}$ with $Q=v(Q)$ and $Q_{2}, \ldots, Q_{g m+1}$ as in the above claim. By the definition of $T(g, n)$, we can find

for which

$$
P_{1}=Q, P_{2}, \ldots, P_{T(g, n)} \in \bar{M}_{g, n}
$$

We then obtain

$$
\left[P_{1}\right]+\cdots+\left[P_{T(g, n)}\right] \in R_{0}\left(\bar{M}_{g, n}\right)
$$

$$
\begin{aligned}
{\left[Q_{1}\right]+\cdots+\left[Q_{g m+1}\right]+\sum_{i=2}^{T(g, n)}(g m+1)\left[\sigma\left(P_{i}\right)\right] } & =(g m+1)[\sigma(Q)]+\sum_{i=2}^{T(g, n)}(g m+1)\left[\sigma\left(P_{i}\right)\right] \\
& =(g m+1) \sigma_{*}\left(\left[P_{1}\right]+\cdots+\left[P_{T(g, n)}\right]\right) \in R_{0}\left(\bar{M}_{g, n+m}\right) .
\end{aligned}
$$

Hence, $T(g, n+m) \leq(g m+1) \cdot T(g, n)$.

We now prove the required claim. For

$$
Q=\left(C, p_{1}, \ldots, p_{n}\right) \in \mathcal{M}_{g, n},
$$

the fibre $v^{-1}(Q)$ is isomorphic to a blow-up of the product $C^{m}$. Since the natural map

$$
v^{-1}(Q) \rightarrow C^{m}
$$

is a birational morphism between nonsingular varieties, we have an induced isomorphism

$$
A_{0}\left(v^{-1}(Q)\right) \rightarrow A_{0}\left(C^{m}\right)
$$

by [Ful98, Example 16.1.11]. We can therefore verify the claim on $C^{m}$ instead of $v^{-1}(Q)$. The image of $\sigma(Q)$ in $C^{m}$ is exactly the point

$$
\left(p_{n}, \ldots, p_{n}\right) \in C^{m}
$$

By Riemann-Roch, every line bundle on $C$ of degree at least $g$ is effective. In other words, any divisor of degree at least $g$ can be written as a sum of points on $C$. Assume we are given

$$
Q_{1}=\left(x_{1}, \ldots, x_{m}\right) \in C^{m} .
$$


Then, there exist points $x_{1,1}, \ldots, x_{1, g} \in C$ satisfying

$$
\left[x_{1,1}\right]+\cdots+\left[x_{1, g}\right]=(g+1)\left[p_{n}\right]-\left[x_{1}\right] \in A_{0}(C) .
$$

Let $Q_{i+1}=\left(x_{1, i}, x_{2}, \ldots, x_{m}\right) \in C^{m}$ for $i=1, \ldots, g$. We have

$$
\left[Q_{1}\right]+\cdots+\left[Q_{g+1}\right]=(g+1)\left[\left(p_{n}, x_{2}, \ldots, x_{m}\right)\right] \in A_{0}\left(C^{m}\right) .
$$

For the next step, there exist points $x_{2,1}, \ldots, x_{2, g} \in C$ satisfying

$$
\left[x_{2,1}\right]+\cdots+\left[x_{2, g}\right]=(2 g+1)\left[p_{n}\right]-(g+1)\left[x_{2}\right] \in A_{0}(C) .
$$

Let $Q_{g+1+i}=\left(p_{n}, x_{2, i}, x_{3}, \ldots, x_{n^{\prime}}\right)$ for $i=1, \ldots, g$. We have

$$
\left[Q_{1}\right]+\cdots+\left[Q_{2 g+1}\right]=(2 g+1)\left[\left(p_{n}, p_{n}, x_{3}, \ldots, x_{n^{\prime}}\right)\right] \in A_{0}\left(C^{m}\right) .
$$

After iterating the above procedure, we find points $Q_{1}, \ldots, Q_{g m+1}$ satisfying

$$
\left[Q_{1}\right]+\cdots+\left[Q_{g m+1}\right]=(g m+1)\left[\left(p_{n}, \ldots, p_{n}\right)\right] \in A_{0}\left(C^{m}\right)
$$

as desired.

Question 7.4. Does $T(g, n)$ really grow linearly as $n \rightarrow \infty$ ?

By results ${ }^{14}$ of Voisin (see Theorem 1.4 of [Voil8]), the analogous $T$ number of an abelian variety $A$ is at least $\operatorname{dim}(A)+1$. The linear growth there perhaps also suggests a linear lower bound for $T(g, n)$ as $n \rightarrow \infty$.

\section{References}

[Be87] A. A. Beilinson, Height pairing between algebraic cycles, $K$-theory, arithmetic and geometry (Moscow, 1984-1986), Lecture Notes in Math., vol. 1289, Springer, Berlin, 1987, pp. 1-25.

[Ben14] L. Benzo, Uniruledness of some moduli spaces of stable pointed curves, J. Pure Appl. Algebra 218 (2014), no. 3, 395-404.

[BF97] K. Behrend and B. Fantechi, The intrinsic normal cone, Invent. Math. 128 (1997), no. 1, 45-88.

[BL00] J. Bryan and N. C. Leung, The enumerative geometry of K3 surfaces and modular forms, J. Amer. Math. Soc. 13 (2000), no. 2, 371-410.

[Blo85] S. Bloch, Algebraic cycles and values of L-functions. II, Duke Math. J. 52 (1985), no. 2, 379-397.

[BP00] M. Boggi and M. Pikaart, Galois covers of moduli of curves, Compositio Math. 120 (2000), no. 2, 171-191.

[BV04] A. Beauville and C. Voisin, On the Chow ring of a K3 surface, J. Algebraic Geom. 13 (2004), no. 3, 417-426.

[BV05] A. Bruno and A. Verra, $\bar{M}_{15}$ is rationally connected, Projective varieties with unexpected properties, Walter de Gruyter, Berlin, 2005, pp. 51-65.

[CDGK20] C. Ciliberto, T. Dedieu, C. Galati, and A. L. Knutsen, A note on Severi varieties of nodal curves on Enriques surfaces, Colombo, Elisabetta (ed.) et al., Birational geometry and moduli spaces. Collected papers presented at the INdAM workshop, Rome, Italy, June 1lâĂŞ15, 2018. Springer INdAM Series 39, 29-36 (2020).

[CF07] G. Casnati and C. Fontanari, On the rationality of moduli spaces of pointed curves, J. Lond. Math. Soc. (2) 75 (2007), no. 3, 582-596.

[Che99] X. Chen, Rational curves on K3 surfaces, J. Algebraic Geom. 8 (1999), no. 2, 245-278.

\footnotetext{
${ }^{14}$ We thank Qizheng Yin for pointing out the connection.
} 
[CJWZ17] E. Clader, F. Janda, X. Wang, and D. Zakharov, Topological recursion relations from Pixton's formula, arXiv preprint arXiv:1704.02011 (2017).

[Far09] G. Farkas, The global geometry of the moduli space of curves, Algebraic geometry-Seattle 2005. Part 1, Proc. Sympos. Pure Math., vol. 80, Amer. Math. Soc., Providence, RI, 2009, pp. 125-147.

[FP05] C. Faber and R. Pandharipande, Relative maps and tautological classes, J. Eur. Math. Soc. (JEMS) 7 (2005), no. 1, 13-49.

[FP13] Tautological and non-tautological cohomology of the moduli space of curves, Handbook of moduli. Vol. I, Adv. Lect. Math. (ALM), vol. 24, Int. Press, Somerville, MA, 2013, pp. 293-330.

[Ful98] W. Fulton, Intersection theory, second ed., Ergebnisse der Mathematik und ihrer Grenzgebiete. 3. Folge. A Series of Modern Surveys in Mathematics, vol. 2, Springer-Verlag, Berlin, 1998.

[FV18] G. Farkas and A. Verra, The universal K3 surface of genus 14 via cubic fourfolds, J. Math. Pures Appl. (9) 111 (2018), 1-20.

[GP99] T. Graber and R. Pandharipande, Localization of virtual classes, Invent. Math. 135 (1999), no. 2, 487-518.

[GP03] _ Constructions of nontautological classes on moduli spaces of curves, Michigan Math. J. 51 (2003), no. 1, 93-109.

[GV01] T. Graber and R. Vakil, On the tautological ring of $\bar{M}_{g, n}$, Turkish J. Math. 25 (2001), no. 1, 237-243.

[Har86] B. Harbourne, The geometry of rational surfaces and Hilbert functions of points in the plane, Proceedings of the 1984 Vancouver conference in algebraic geometry, CMS Conf. Proc., vol. 6, Amer. Math. Soc., Providence, RI, 1986, pp. 95-111.

[Hir89] A. Hirschowitz, Une conjecture pour la cohomologie des diviseurs sur les surfaces rationnelles génériques, J. Reine Angew. Math. 397 (1989), 208-213.

[HL97] R. Hain and E. Looijenga, Mapping class groups and moduli spaces of curves, Algebraic geometrySanta Cruz 1995, Proc. Sympos. Pure Math., vol. 62, Amer. Math. Soc., Providence, RI, 1997, pp. 97-142.

[Huy16] D. Huybrechts, Lectures on K3 surfaces, Cambridge Studies in Advanced Mathematics, vol. 158, Cambridge University Press, Cambridge, 2016.

[Jan90] U. Jannsen, Mixed motives and algebraic K-theory, Lecture Notes in Mathematics, vol. 1400, SpringerVerlag, Berlin, 1990, With appendices by S. Bloch and C. Schoen.

[Log03] A. Logan, The Kodaira dimension of moduli spaces of curves with marked points, Amer. J. Math. 125 (2003), no. 1, 105-138.

[MP13] D. Maulik and R. Pandharipande, Gromov-Witten theory and Noether-Lefschetz theory, A celebration of algebraic geometry, Clay Math. Proc., vol. 18, Amer. Math. Soc., Providence, RI, 2013, pp. 469-507.

[Muk96] S. Mukai, Curves and K3 surfaces of genus eleven, Moduli of vector bundles (Sanda, 1994; Kyoto, 1994), Lecture Notes in Pure and Appl. Math., vol. 179, Dekker, New York, 1996, pp. 189-197.

[Mum68] D. Mumford, Rational equivalence of 0 -cycles on surfaces, J. Math. Kyoto Univ. 9 (1968), 195-204.

[Pan18] R. Pandharipande, A calculus for the moduli space of curves, Algebraic geometry: Salt Lake City 2015, Proc. Sympos. Pure Math., vol. 97, Amer. Math. Soc., Providence, RI, 2018, pp. 459-487.

[PY20] R. Pandharipande and Q. Yin, Relations in the tautological ring of the moduli space of K3 surfaces, J. Eur. Math. Soc. (JEMS) 22 (2020), no. 1, 213-252. 
[Ro72] A. A. Roŭtman, Rational equivalence of zero-dimensional cycles, Mat. Sb. (N.S.) 89(131) (1972), 569-585, 671.

[Sch18] I. Schwarz, On the Kodaira dimension of $\overline{\mathcal{N}}_{g, n}$, arXiv e-prints (2018), arXiv:1811.01193.

[Sri87] V. Srinivas, Rational equivalence of 0 -cycles on normal varieties over $\mathbf{C}$, Algebraic geometry, Bowdoin, 1985 (Brunswick, Maine, 1985), Proc. Sympos. Pure Math., vol. 46, Amer. Math. Soc., Providence, RI, 1987, pp. 475-482.

[Sv18] J. Schmitt and J. van Zelm, Intersections of loci of admissible covers with tautological classes, arXiv e-prints (2018), arXiv:1808.05817.

[SY] J. Schmitt and Q. Yin, Effectivity degree of zero cycles, in preparation.

[Ver05] A. Verra, The unirationality of the moduli spaces of curves of genus 14 or lower, Compos. Math. 141 (2005), no. 6, 1425-1444.

[Voi15] C. Voisin, Unirational threefolds with no universal codimension 2 cycle, Invent. Math. 201 (2015), no. 1, 207-237.

[Voi18] _ Chow ring and gonality of general abelian varieties, Annales Henri Lebesgue 1 (2018), 313-332. 